Research Article

\title{
Health-Related Quality of Life and Its Influencing Factors in Patients with Hepatitis B: A Cross-Sectional Assessment in Southeastern China
}

\author{
Ping Chen, ${ }^{1,2}$ Fen Zhang, ${ }^{3}$ Yiqun Shen, ${ }^{4}$ Yubo Cai, ${ }^{5}$ Chaolei Jin, ${ }^{6}$ Yan Li, ${ }^{7}$ Mingmin Tu, ${ }^{8}$ \\ Weizhen Zhang, ${ }^{9}$ Yu Wang, ${ }^{10}$ Shi-Feng Zhang, ${ }^{11}$ Jiangyun Wang, ${ }^{12}$ and Lanjuan Li $\mathbb{D}^{3}$ \\ ${ }^{1}$ Department of Infectious Diseases, Shulan (Hangzhou) Hospital Affiliated to Zhejiang Shuren University Shulan \\ International Medical College, Hangzhou 310012, China \\ ${ }^{2}$ Department of Infectious Diseases, Zhengzhou Cancer Hospital of Henan University, Zhengzhou 450000, China \\ ${ }^{3}$ State Key Laboratory for Diagnosis and Treatment of Infectious Diseases, National Clinical Research \\ Center for Infectious Diseases, Collaborative Innovation Center for Diagnosis and Treatment of Infectious Diseases, \\ The First Affiliated Hospital, College of Medicine, Zhejiang University, Hangzhou 310003, China \\ ${ }^{4}$ Huzhou Central Hospital, Affiliated Central Hospital Huzhou University, HuZhou 313000, China \\ ${ }^{5}$ School of Medicine, Zhejiang University, Hangzhou 310058, China \\ ${ }^{6}$ School of Clinical Medicine, Henan University, Kaifeng 475000, China \\ ${ }^{7}$ The Academician Workstation, Zhengzhou Cancer Hospital of Henan University, Zhengzhou 450000, China \\ ${ }^{8}$ Hangzhou Tongchuang Medical Laboratory, Hangzhou 310012, China \\ ${ }^{9}$ Department of Oncology, Zhengzhou Cancer Hospital of Henan University, Zhengzhou 450000, China \\ ${ }^{10}$ Department of Hematology, Zhengzhou Cancer Hospital of Henan University, Zhengzhou 450000, China \\ ${ }^{11}$ Department of Stomatology, Zhengzhou Cancer Hospital of Henan University, Zhengzhou 450000, China \\ ${ }^{12}$ Department of Cosmetic Surgery, Zhengzhou Cancer Hospital of Henan University, Zhengzhou 450000, China
}

Correspondence should be addressed to Lanjuan Li; ljli@zju.edu.cn

Received 19 March 2021; Accepted 27 June 2021; Published 7 July 2021

Academic Editor: Yu-Chen Fan

Copyright (c) 2021 Ping Chen et al. This is an open access article distributed under the Creative Commons Attribution License, which permits unrestricted use, distribution, and reproduction in any medium, provided the original work is properly cited.

Health-related quality of life (HRQoL) is an important aspect in the management of patients with hepatitis B (HB), which remains a serious health problem in China. There have been relatively few HRQoL studies involving Chinese patients with HB. The aim of this study was to analyze HRQoL in patients diagnosed with HB living in Zhejiang Province, China. A cross-sectional sample of 98 patients with chronic $\mathrm{HB}(\mathrm{CHB}), 56$ patients with advanced $\mathrm{HB}$ that have developed cirrhosis, and 48 healthy controls (HCs), all from Zhejiang Province, was used in this study. HRQoL was assessed using Short-Form 36 (SF-36) version 2, European quality of life questionnaire-5 dimensions (EQ-5D), and chronic liver disease questionnaire (CLDQ). Intergroup score differences were detected with $U$ tests. Factors with a significant effect on HRQoL were identified with Spearman correlational analyses. Patients with HB (both groups) had lower SF-36 scores than HCs $(p<0.01)$, with the exception of general health subscores. Patients with $\mathrm{HB}$ cirrhosis had the lowest scores in the EQ-5D visual analog scale (VAS) component. Furthermore, patients with HB cirrhosis had lower $(p<0.01)$ CLDQ scores than patients with CHB. In our HB patient cohort, disease stage and income level were the factors most associated with HRQoL variables; age, education level, and marital status were, each, also significantly associated with some HRQoL variables in patients with HB in our study $(p<0.05$ or $p<0.01)$. HRQoL is diminished in patients with HB in southeastern China. Disease stage and income emerged as key determinants of HRQoL scores. Augmenting social and medical supports for patients with $\mathrm{HB}$, especially those with a socioeconomic status and an advanced disease stage, may help to enhance HRQoL. 


\section{Introduction}

Hepatitis $B(\mathrm{HB})$ is a serious and incurable disease caused by infection with $\mathrm{HB}$ virus (HBV) [1]. HBV infection can be confirmed with $\mathrm{HB}$ surface antigen (HBsAg) testing. According to a recent global hepatitis report produced by the World Health Organization, 240 million individuals were HBsAg-positive worldwide in 2017, including more than 74 million in China alone [2,3]. Hence, the geographical distribution of people infected with the HBV is quite unbalanced, with the largest portion of affected people living in China [4,5]. Additionally, of the approximately 850,000 US citizens and residents reported in 2012 to have been diagnosed with chronic $\mathrm{HB}(\mathrm{CHB})$, nearly half were of nonHispanic Asian descent [4].

Newborn HBV vaccination was introduced in China in 1992 [6-8]. In 1992, nearly one in ten people in the mainland Chinese population (9.75\%) were HBsAg-positive; by 2006, after 14 years of intensive vaccination, the HBsAg-positive rate had dropped to $7.18 \%$ [6]. Hence, though infection rates are improving, the $\mathrm{HBV}$-infected population in China remains fairly high, and $\mathrm{HBV}$ is an ongoing public health challenge. It has been suggested that vaccination schedule delays are an important factor in the slow progress in HBVinfection rate reduction in China [9].

Patients with $\mathrm{CHB}$ exhibit a variety of disease processes, including liver decompensation, cirrhosis, and even primary hepatocellular carcinoma, which can severely affect the quality of life and life expectancy. The Global Health Data Exchange data for 2017 showed a loss of about 21 million disability-adjusted life-years due to HB-related morbidity [10]. Although clinically available antiviral treatments can delay $\mathrm{CHB}$ disease progression and improve outcomes for patients diagnosed with $\mathrm{CHB}$, high treatment costs can have severe degradative effects on patients' quality of life [11], with more than 10,000 Yuan per year being spent on drugs and routine examinations for each patient in China [10]. Since chronic hepatitis $\mathrm{C}$ has been shown to affect health-related quality of life (HRQoL) [12,13], there has been an increasing interest in examining the impacts of $\mathrm{HB}$ on HRQoL, with numerous studies exploring the relationship between $\mathrm{HB}$ and HRQoL in patients living in the USA, Vietnam, Singapore, and Hong Kong having been published [10,14-16]. Data describing HRQoL among patients with $\mathrm{HB}$ in China remain scarce, especially for patients in Zhejiang Province.

In this study, we evaluated the HRQoL of patients diagnosed with $\mathrm{HB}$ in southeastern China, including patients with $\mathrm{CHB}$ as well as patients with cirrhosis secondary to HBV infection. HRQoL was assessed with the Short-Form 36 (SF-36) version 2, the European quality of life questionnaire5 dimensions (EQ-5D), and the chronic liver disease questionnaire (CLDQ); and the mood (including depressive symptoms) of the patients was further assessed with the SF36 and EQ-5D. Potential HRQoL-influencing factors in the study population were assessed with a multivariate correlational analysis. The present study is intended to produce data that can improve our understanding of HRQoL among patients with $\mathrm{HB}$ in southeastern China and thus provide guidance for improving the provision of health services and timely interventions for this patient population.

\section{Respondents and Methods}

2.1. Study Population. This project was funded by the MegaProject for National Science and Technology Development (as part of the $12^{\text {th }}$ five-year plan for China) and the Department of Health of Zhejiang Province. This study was carried out in 2013 principally in Huzhou City in the northern region of Zhejiang Province, which is located in southeastern China. Huzhou City is of median economic development for Zhejiang Province with a relatively small transient population. We invited the 300 patients with $\mathrm{CHB}$ who have been receiving $\mathrm{CHB}$ management at Huzhou Central since 2008, including taking antiviral drugs, to participate in this study. Forty-eight HCs were recruited from patients receiving their regular annual physical examinations at Huzhou Central Hospital. All potential participants were asked to sign written informed consent forms. This study was conducted in compliance with the principles of the 1975 Declaration of Helsinki and was approved by the Ethics Committee of the First Affiliated Hospital, Zhejiang University.

$\mathrm{CHB}$ diagnoses were made in accordance with the standards established in 2000 by the Chinese National Conference on Viral Hepatitis in Xi'an. Cirrhosis due to HB was diagnosed based on liver biopsy (performed for medical reasons, not for this study) and ultrasonographic evidence of ascites, gastroesophageal varices, hepatic decompensation changes, and/or hepatic encephalopathy. The inclusion criteria for patients with $\mathrm{HB}$ were as follows: a diagnosis of $\mathrm{CHB}$ without cirrhosis ( $\mathrm{CHB}$ group) or with cirrhosis ( $\mathrm{HB}$ cirrhosis group); currently receiving antiviral therapy; willingness to provide informed consent for participation [17]. The exclusion criteria for patients with $\mathrm{HB}$ were discontinuation of antiviral therapy; any chronic disease comorbidity; previous or current history of mental illness. The inclusion criteria for HCs were as follows: age in the range of 18-80 years and willingness to provide informed consent for participation [17]. The exclusion criteria for HCs were any chronic disease diagnosis and previous or current history of mental illness.

The 300 potential patients with $\mathrm{HB}$ invited to participate in this study all had complete diagnostic records. Of these 300 patients, 146 patients were excluded for not receiving antiviral therapy, leaving 164 patients with HB (see Supplementary Figure 1 for patient enrollment flow summary). All of the $48 \mathrm{HCs}$ recruited met the inclusion criteria and none were excluded due to the exclusion criteria. Thus, we administered questionnaires to the remaining 164 patients and $48 \mathrm{HCs} ; 10$ patients returned incomplete questionnaires and were thus not included in the final analyses. Thus, 154 patients with $\mathrm{HB}$ and $48 \mathrm{HCs}$ were included in our final analyses.

2.2. Interview Process. Participants' HRQoL levels were assessed by trained interviewers using the SF-36 version 2, 
EQ-5D, and CLDQ [18]. Simultaneously, a self-designed questionnaire was administered to acquire each participant's sociodemographic information [19-21], including gender, age, education level, occupation, household monthly income, course of illness, current health status, cognitive recognition of $\mathrm{HB}$, and medicinal treatment compliance. Cognitive recognition of $\mathrm{HB}$ was evaluated with nine yes-no questions ( 1 point per question) based on the recommendations of Gallegos-Orozco et al. [22,23] such that scores $\leq 6$ points indicated a lack of disease recognition, 7 points indicated fair recognition, and 8 points represented good or better recognition. Medicinal compliance was evaluated with six yes-no questions (1 point per question), with scores $\geq 4$ considered to indicate satisfactory compliance and scores $\leq 1$ indicated poor compliance.

The SF-36 instrument consists of 36 items encompassing the following eight domains [24]: physical function, role physical, bodily pain, general health, role emotional, social function, vitality, and mental health. The first four-dimension subscores were summed to produce a physical status component summary (PCS) score; the latter four-dimension subscores were summed to produce a mental health component summary (MCS) score. These two summary scores were then added to yield an overall HRQoL score, with higher scores indicating an overall better HRQoL.

The EQ-5D questionnaire [24] consists of a health descriptive component and a visual analog scale (VAS) component. The health descriptive component has five dimensions (mobility, self-care, usual activities, pain/discomfort, and anxiety/depression); each dimension was graded according to three levels of severity. We calculated overall EQ-5D-3L scores according to the UK TTO value set (range: -0.594 to 1.0 ). The EQ-5D VAS is a $20-\mathrm{cm}$-long, 0-100-point scale, wherein scores indicate health status from the worst imaginable state (score of 0 ) to the best imaginable state (score of 100).

The CLDQ is a 29-item liver-disease-specific scale composed of six dimensions [25]: abdominal symptoms, fatigue, systemic symptoms, activity, emotional function, and worry. The total CLDQ score is an average of the sixdimensional scores, in which higher scores indicate a better quality of life.

2.3. Data Analysis. Pearson's chi-square tests were used to compare sociodemographic variables between $\mathrm{HB}$ subjects and HCs. Because a normal distribution test indicated that the data were nonparametric, Kruskal-Wallis tests were used adopted to compare sociodemographic variables among the three groups (CHB, HB cirrhosis, and $\mathrm{HC}$ ), and Mann-Whitney $U$ tests were used to compare SF-36, EQ$5 \mathrm{D}$, and CLDQ scores among the three groups and between the two HB groups. Spearman correlation values were calculated to identify independent factors that are significantly related to HRQoL. The raw data were analyzed in SPSS 25.0 with a significance criterion of $p<0.05$. Mean $(M)$ values are reported with standard deviations (SDs) and 95\% confidence intervals (CIs); median values are reported with interquartile ranges.

\section{Results}

3.1. Study Group Characteristics. The majority of our sample of patients diagnosed with $\mathrm{HB}$ was males (69.5\%), with a mean age of $50.59 \pm 10.31$ years and a mean income level above 1500 per month per family. The sociodemographic characteristics of each group are summarized in Table 1 (and the patients' biochemical blood test results are summarized by group in Supplementary Table 1). Notably, compared with HCs, the CHB group was more likely to be older, males, and nonprofessionals and had a lower income. Patient compliance levels were fair, with about $85 \%$ of the patients complying with their doctors' instructions overall. The majority of patients with HB had a good cognitive understanding of their disease, while only about one in seven patients with $\mathrm{HB}$ had a poor understanding. The HB cirrhosis group exhibited significantly better treatment compliance than the CHB group $(p<0.05)$. Nearly $30 \%$ of patients in the $\mathrm{HB}$ cirrhosis group reported that they were less than two years into their disease course, which suggests that many may have been unaware of their infection and health status for a substantial period of time (Table 1).

\section{2. $H R Q o L$}

3.2.1. SF-36. Compared with HCs, the CHB group had a significantly lower physical function, role physical, role emotional, social function, vitality, and mental health subscores as well as a lower MCS score (Table 2). Meanwhile, compared with HCs, the HB cirrhosis group had a lower physical function, role physical, bodily pain, role emotional, social function, and vitality subscores as well as lower MCS and PCS scores (Table 2). Conversely, compared with the $\mathrm{HC}$ group, the $\mathrm{CHB}$ and $\mathrm{HB}$ cirrhosis groups had higher general health subscores (Figure 1 and Table 2). Compared with the CHB group, the HB cirrhosis group had significantly lower PCS scores but significantly higher physical functioning and role physical subscores (Figure 1 and Table 2).

3.2.2. EQ-5D. There were no significant differences among the three groups in the health descriptive component of the EQ-5D (Table 3). However, patients with HB cirrhosis had slightly lower VAS scores than patients with CHB (75 vs. 79) and markedly lower VAS scores than the HC group (75 vs. 83) (Figure 2).

3.2.3. CLDQ. The $\mathrm{HB}$ cirrhosis group had significantly lower CLDQ scores than the CHB group in the abdominal symptoms, systemic symptoms, activity, and executive function domains of the CLDQ (all $p<0.05$; Table 4 and Figure 3). 
TABLE 1: Sociodemographic characteristics and clinical parameters of the respondents (because some respondents did not answer all questions, the numbers of valid responses for characteristics differ).

\begin{tabular}{|c|c|c|c|c|c|c|c|}
\hline \multirow{2}{*}{ Characteristic } & & \multicolumn{2}{|c|}{$\mathrm{HC}$} & \multicolumn{2}{|c|}{$\mathrm{CHB}$} & \multicolumn{2}{|c|}{ HB cirrhosis } \\
\hline & & $N$ & $\%$ & $N$ & $\%$ & $N$ & $\%$ \\
\hline \multirow{2}{*}{ Gender** } & Male & 22 & 50.0 & 68 & 69.4 & 39 & 69.6 \\
\hline & Female & 22 & 50.0 & 30 & 30.6 & 17 & 30.4 \\
\hline \multirow{4}{*}{ Age $^{* * \# \#}$} & $<40$ years & 29 & 64.4 & 20 & 20.4 & 3 & 5.4 \\
\hline & $40-50$ years & 10 & 22.2 & 37 & 37.8 & 15 & 26.8 \\
\hline & $50-60$ years & 2 & 4.4 & 28 & 28.6 & 28 & 50.0 \\
\hline & $\geq 60$ years & 4 & 8.9 & 13 & 13.3 & 10 & 17.9 \\
\hline \multirow{3}{*}{ Education**\#\# } & Elementary school or less & 2 & 4.3 & 45 & 45.9 & 35 & 62.5 \\
\hline & Middle/high school & 27 & 58.7 & 48 & 49.0 & 21 & 37.5 \\
\hline & College/postgraduate & 17 & 37.0 & 5 & 5.1 & 0 & 0.0 \\
\hline \multirow{3}{*}{ Marital status** } & Unmarried & 13 & 28.3 & 6 & 6.1 & 0 & 0.0 \\
\hline & Married & 28 & 60.9 & 86 & 87.8 & 52 & 92.9 \\
\hline & Separated & 5 & 10.9 & 6 & 6.1 & 4 & 7.1 \\
\hline \multirow{3}{*}{ Household monthly income/person**\# } & $<1500$ yuan & 4 & 9.1 & 28 & 28.6 & 21 & 37.5 \\
\hline & $1500-3000$ yuan & 3 & 6.8 & 27 & 27.6 & 17 & 30.4 \\
\hline & $\geq 3000$ yuan & 37 & 84.1 & 43 & 43.9 & 18 & 32.1 \\
\hline \multirow{3}{*}{ Compliance $^{\#}$} & Poor & - & - & 13 & 13.4 & 1 & 1.9 \\
\hline & Medium & - & - & 20 & 20.6 & 14 & 25.9 \\
\hline & Good & - & - & 64 & 66.0 & 39 & 72.2 \\
\hline \multirow{3}{*}{ Cognitive recognition of disease } & Low & - & - & 14 & 14.3 & 8 & 14.3 \\
\hline & Medium & - & - & 22 & 22.4 & 17 & 30.4 \\
\hline & High & - & - & 62 & 63.3 & 31 & 55.4 \\
\hline \multirow{3}{*}{ Disease course } & $\leq 2$ years & - & - & 28 & 28.6 & 16 & 29.1 \\
\hline & $3-7$ years & - & - & 34 & 34.7 & 21 & 38.2 \\
\hline & $\geq 7$ years & - & - & 36 & 36.7 & 18 & 32.7 \\
\hline
\end{tabular}

${ }^{*} p<0.05$ and $^{* *} p<0.01 \mathrm{HB}$ (both groups) vs. HCs. ${ }^{\#} p<0.05$ and ${ }^{\# \#} p<0.01 \mathrm{CHB}$ vs. HB cirrhosis.

TABLE 2: Comparison of SF-36 results by group.

\begin{tabular}{lcccccc}
\hline \multirow{2}{*}{ SF-36 domain or section summary } & \multicolumn{2}{c}{ HC } & \multicolumn{2}{c}{ CHB } & \multicolumn{2}{c}{ HB cirrhosis } \\
& Mean (SD) & 95\% CI & Mean (SD) & 95\% CI & Mean (SD) & 95\% CI \\
\hline Physical function & $92.16(1.7)$ & $88.74-95.57$ & $84.37(1.52)^{* *}$ & $81.35-87.39$ & $77.35(2.1)^{* * \# \#}$ & $73.14-81.56$ \\
Role physical & $76.47(5.3)$ & $65.82-87.13$ & $40.1(4.51)^{* *}$ & $31.15-49.06$ & $12.95(3.98)^{* * \# \#}$ & $4.97-20.92$ \\
Bodily pain & $88.39(2.66)$ & $83.04-93.74$ & $83.57(1.86)$ & $79.87-87.27$ & $79.21(2.64)^{* *}$ & $73.93-84.5$ \\
General health & $29.41(1.97)$ & $25.44-33.37$ & $49.29(1.41)^{* *}$ & $46.49-52.09$ & $46.34(1.93)^{* *}$ & $42.47-50.2$ \\
PCS & $71.06(2.69)$ & $65.63-76.48$ & $63.89(1.91)$ & $60.1-67.68$ & $53.96(1.89)^{* * \# \#}$ & $50.17-57.75$ \\
Role emotional & $80.95(4.66)$ & $71.58-90.33$ & $55.56(4.75)^{* *}$ & $46.12-64.99$ & $48.81(6.24)^{* *}$ & $36.3-61.31$ \\
Social function & $82.57(3.05)$ & $76.44-88.7$ & $73.24(2.4)^{* *}$ & $68.48-78$ & $73.21(2.97)^{* *}$ & $67.27-79.16$ \\
Vitality & $70.92(2.84)$ & $65.21-76.62$ & $62.85(1.78)^{*}$ & $59.31-66.39$ & $62.23(2.63)^{*}$ & $56.97-67.5$ \\
Mental health & $71.67(2.23)$ & $67.19-76.15$ & $65.8(1.72)^{*}$ & $62.39-69.22$ & $65.86(2.56)$ & $60.72-70.99$ \\
MCS & $78.14(2.57)$ & $72.96-83.33$ & $64.49(2.24)^{* *}$ & $60.05-68.93$ & $62.53(3.15)^{* *}$ & $56.22-68.83$ \\
\hline
\end{tabular}

${ }^{*} p<0.05$ and ${ }^{* *} p<0.01$ vs. HC; ${ }^{\# \#} p<0.01$ vs. CHB.

3.3. Correlation Analysis. Correlation analyses examining which participant characteristics are associated with HRQoL-representative variables (PCS score, MCS score, SF36 total score, EQ-D5 VAS score, and CLDQ score) showed that the disease stage, age, education, marital status, and household income were significantly associated with HRQoL. Furthermore, disease stage and household income appeared to be the factors that were most strongly related to HRQoL (Table 5).

Because some respondents did not answer all questions, the number of valid responses for each characteristic may vary.

\section{Discussion}

In this study, we observed broadly lower HRQoL scores in patients with $\mathrm{HB}$, especially patients with $\mathrm{HB}$ cirrhosis, compared with HCs. However, the patients with HB, surprisingly, had higher general health SF-36 subscores than HCs in our study. This general health finding is inconsistent with the results of several previous reports showing an opposite trend [16,24,26] Meanwhile, Thuluvath et al. [13] and Helbling et al. [11] found no significant difference in SF36 general health subscores between patients with $\mathrm{HB}$ and HCs. 


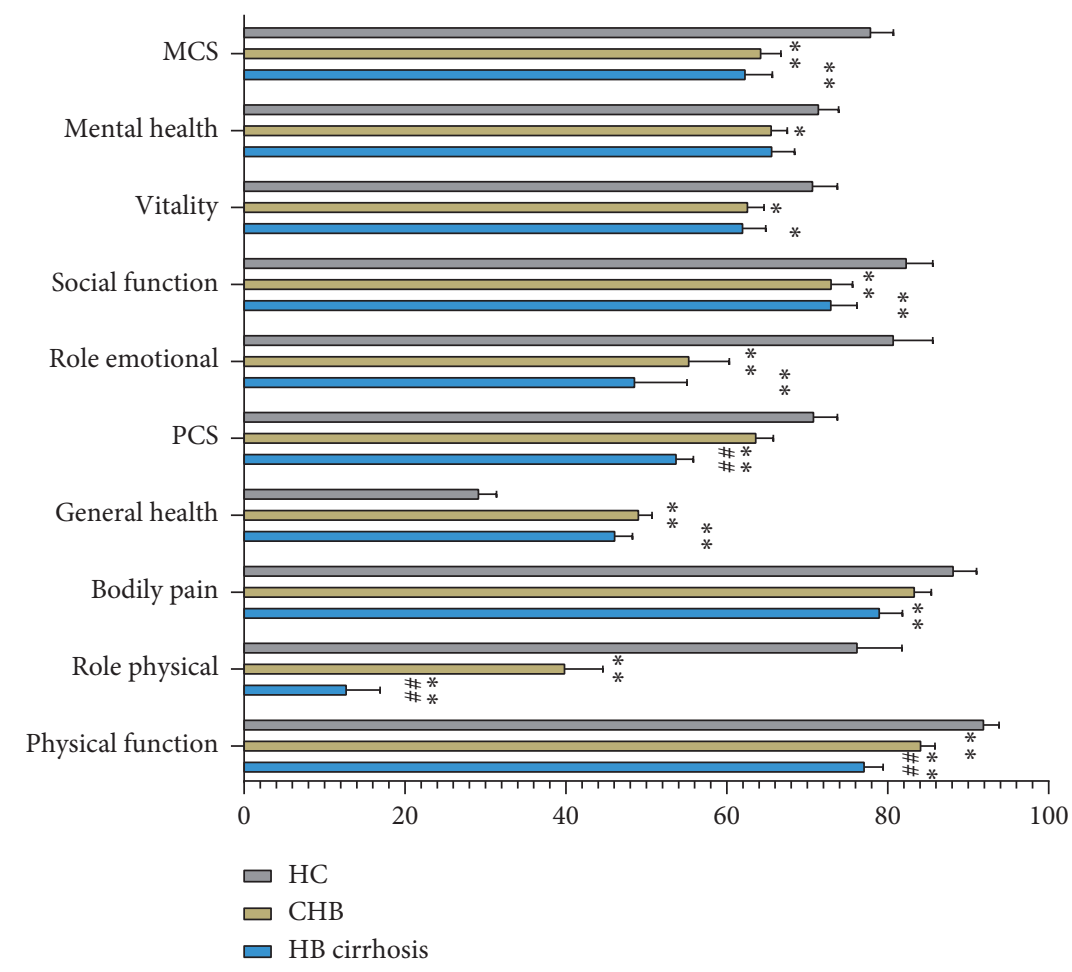

Figure 1: Comparison of SF-36 results across study groups. Note that compared with HCs, the HB cirrhosis group had significantly reduced scores for all dimensions, except for mental health, and the CHB group had significantly reduced scores for all dimensions except for bodily pain and PCS scores. The two HB groups only differed from one another in the role physical and PCS scores. ${ }^{*} p<0.05$ and ${ }^{* *} p<0.01$ vs. HC. ${ }^{\#} p<0.05$ and ${ }^{\# \#} p<0.01$ vs. CHB.

TABle 3: Comparison of EQ-5D dimension score levels and VAS scores between study groups.

\begin{tabular}{|c|c|c|c|c|c|c|c|}
\hline \multirow{2}{*}{$\begin{array}{l}\text { Component } \\
\text { Dimension: level }\end{array}$} & & \multicolumn{2}{|c|}{$\mathrm{HC}$} & \multicolumn{2}{|c|}{$\mathrm{CHB}$} & \multicolumn{2}{|c|}{ HB cirrhosis } \\
\hline & & $N / M$ & $\% / 95 \% \mathrm{CI}$ & $N / M$ & $\% / 95 \% \mathrm{CI}$ & $N / M$ & $\% / 95 \%$ CI \\
\hline \multirow{3}{*}{ Mobility } & Good & 46 & 95.8 & 96 & 98.0 & 54 & 96.4 \\
\hline & Medium & 2 & 4.2 & 2 & 2.0 & 2 & 3.6 \\
\hline & Serious & 0 & 0.0 & 0 & 0.0 & 0 & 0.0 \\
\hline \multirow{3}{*}{ Self-care } & Good & 46 & 95.8 & 96 & 98.0 & 56 & 100.0 \\
\hline & Medium & 2 & 4.2 & 2 & 2.0 & 0 & 0.0 \\
\hline & Serious & 0 & 0.0 & 0 & 0.0 & 0 & 0.0 \\
\hline \multirow{3}{*}{ Usual activities } & Good & 45 & 93.8 & 91 & 92.9 & 52 & 92.9 \\
\hline & Medium & 3 & 6.3 & 6 & 6.1 & 4 & 7.1 \\
\hline & Serious & 0 & 1.0 & 1 & 1.0 & 0 & 0.0 \\
\hline \multirow{3}{*}{ Pain/discomfort } & Good & 41 & 85.4 & 77 & 79.4 & 44 & 78.6 \\
\hline & Medium & 6 & 12.5 & 20 & 20.6 & 12 & 21.4 \\
\hline & Serious & 1 & 2.1 & 0 & 0.0 & 0 & 0.0 \\
\hline \multirow{3}{*}{ Anxiety/depression } & Good & 38 & 80.9 & 76 & 78.4 & 41 & 73.2 \\
\hline & Medium & 7 & 14.9 & 20 & 20.6 & 14 & 25.0 \\
\hline & Serious & 2 & 4.3 & 1 & 1.0 & 1 & 1.8 \\
\hline \multirow[t]{2}{*}{ VAS } & & $48(84)$ & $80-87$ & $98(79)$ & $77-82$ & $56(75)^{* * \#}$ & $71-78$ \\
\hline & & Mean & SD & Mean & $\mathrm{SD}$ & Mean & $\mathrm{SD}$ \\
\hline EQ-5D-3L & & 0.94 & 0.12 & 0.92 & 0.13 & 0.92 & 0.15 \\
\hline
\end{tabular}

${ }^{* *} p<0.01$ vs. HC; ${ }^{\#} p<0.05$ vs. CHB.

Our findings of lower MCS scores for the CHB and $\mathrm{HB}$ cirrhosis groups, compared with HCs, suggest that the mental health of patients with $\mathrm{HB}$ should be further considered. Although the prevalence of major depressive disorder in China is only $1.5 \%$, the prevalence of major depressive disorder among Chinese patients with $\mathrm{HB}$ has 


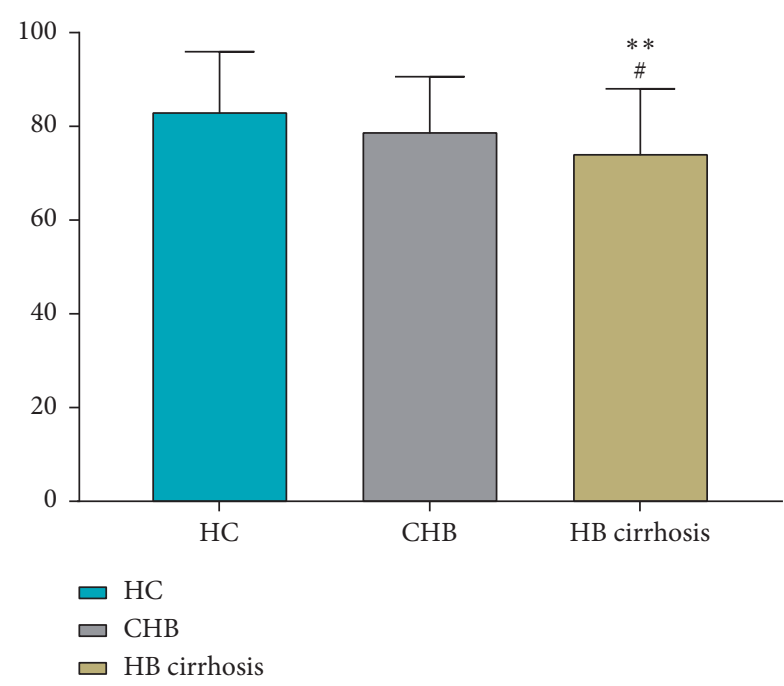

FIGURE 2: Comparison of EQ-5D VAS scores between study groups. The HB cirrhosis groups had significantly $\left({ }^{* *} p<0.01\right)$ lower VAS scores than the HC group and significantly $\left({ }^{*} p<0.05\right)$ lower VAS scores than the CHB group.

TABLE 4: Comparison of CLDQ dimension and total scores between HB groups.

\begin{tabular}{|c|c|c|c|c|}
\hline \multirow{2}{*}{ Dimension } & \multicolumn{2}{|c|}{$\mathrm{CHB}$} & \multicolumn{2}{|c|}{ HB cirrhosis } \\
\hline & mean $(\mathrm{SD})$ & $95 \% \mathrm{CI}$ & mean (SD) & $95 \%$ CI \\
\hline $\begin{array}{l}\text { Abdominal } \\
\text { symptoms }\end{array}$ & $5.95(1.25)$ & $5.7-6.2$ & $5.49(1.25)^{\# \#}$ & $5.15-5.82$ \\
\hline Fatigue & $5.17(1.24)$ & $4.92-5.42$ & $4.82(1.24)$ & $4.48-5.15$ \\
\hline $\begin{array}{l}\text { Systemic } \\
\text { symptoms }\end{array}$ & $5.88(1.07)$ & $5.67-6.1$ & $5.53(1.09)^{\#}$ & $5.24-5.82$ \\
\hline Activity & $5.67(1.18)$ & $5.44-5.91$ & $5.15(1.21)^{\# \#}$ & $4.82-5.47$ \\
\hline $\begin{array}{l}\text { Emotional } \\
\text { function }\end{array}$ & $5.4(1.22)$ & $5.15-5.65$ & $4.98(1.29)^{\#}$ & $4.64-5.33$ \\
\hline Worry & $5.29(1.34)$ & $5.02-5.56$ & $4.89(1.57)$ & $4.46-5.31$ \\
\hline $\begin{array}{l}\text { All dimensions } \\
\text { averaged }\end{array}$ & $5.56(1.05)$ & $5.35-5.77$ & $5.14(1.06)^{\# \#}$ & $4.86-5.43$ \\
\hline
\end{tabular}

been reported to be $6.4 \%[27,28]$. In addition to the physical challenges faced by patients with $\mathrm{HB}$, which may include fatigue, appetite loss, and abdominal pain, the disease itself may result in patients having to endure isolation and even discrimination. In this context, it has been suggested that poor self-awareness may contribute to depression in patients suffering from $\mathrm{CHB}[28]$.

The present EQ-5D results showing significant intergroup differences for only the VAS component of the instrument are in line with Ong et al.'s findings [16]. Patients with $\mathrm{HB}$ cirrhosis had lower CLDQ scores for most of the dimensions, with the exceptions being the fatigue and worry dimensions. Mental health variable scores were similar between the $\mathrm{CHB}$ and $\mathrm{HB}$ cirrhosis groups, although the latter group had significantly worse bodily pain and PCS scores on the SF-36 than HCs (Table 2), while the CHB group did not-they would be expected to experience a greater hardship as a result of their condition. The lack of a significant difference between these two HB groups with

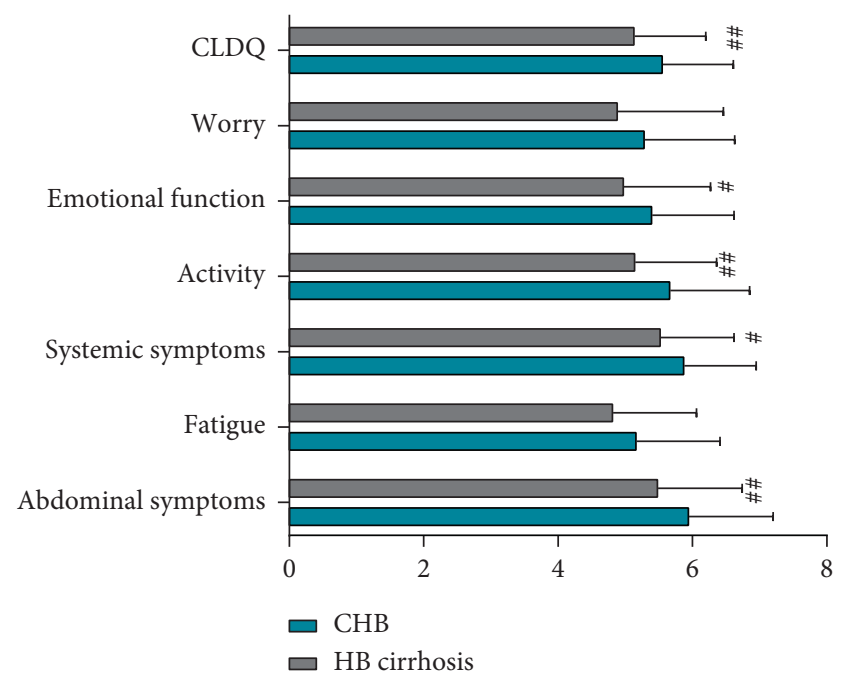

Figure 3: Comparison of CLDQ scores between HB groups. Compared with the $\mathrm{CHB}$ group, the $\mathrm{HB}$ cirrhosis group had significantly worse $\left({ }^{\#} p<0.05\right)$ emotional function and systemic symptom scores as well as significantly worse $\left({ }^{\#} p<0.05\right)$ total CLDQ, activity, and abdominal symptom scores.

respect to mental health could reflect some level of adaptation to the diminished state in patients with HB cirrhosis, including having reduced expectations regarding the quality of life $[14,29]$. Based on these results, it is our view that patients with HBV do not often receive sufficient physical and mental health support.

Our correlational analysis performed to identify HRQoL determinants in patients with $\mathrm{HB}$ showed that HRQoL was inversely related to an advanced disease stage, older age, being without a life partner (unmarried or separated), low education, and lower income levels, consistent with the findings of several prior studies $[10,12,15,29]$. The variables of disease stage and household monthly income emerged as the factors most strongly related to diminution of HRQoL. Thus, our data suggest that these two factors may be key influencing factors of HRQoL. Because household income is related to many other sociodemographic variables, including occupation, education level, and marital status, as well as one's understanding of their disease, it may be a useful composite index for HRQoL in patients suffering from a chronic disease, such as HB. Parkash et al. have argued that a low income status due to a limited education is likely an important reason for delays in patients receiving disease diagnoses and treatment; such delays, consequently, result in patients' disease conditions having a more profoundly negative influence on the quality of life [30]. These findings underscore the need for economic analyses of newly approved treatment regimens and those in the clinical trial pipeline, as well as the need for the development of costeffective strategies. Additionally, our findings indicate that there are unmet needs with respect to targeted services and timely clinical interventions for $\mathrm{HB}$ patient populations at a high risk of a low HRQoL.

The current study had several noteworthy limitations. First, we had a relatively small sample size, which increases 
TABLE 5: Correlation analysis of sociodemographic factor variable influences on HRQoL outcome variables.

\begin{tabular}{|c|c|c|c|c|c|c|}
\hline \multirow{2}{*}{ HRQoL outcome variable } & \multicolumn{6}{|c|}{ Correlated sociodemographic factor, correlation index (4) } \\
\hline & Disease stage & Gender & Age & Education & Marital status & Household monthly income \\
\hline SF-36 & $-0.322^{* *}$ & -0.076 & $-0.331^{* *}$ & $0.231^{* *}$ & $-0.190^{* *}$ & $0.394^{* *}$ \\
\hline PCS & $\{195\}$ & $\{190\}$ & $\{192\}$ & $\{192\}$ & $\{194\}$ & $\{190\}$ \\
\hline SF-36 & $-0.234^{* *}$ & -0.067 & $-0.172^{*}$ & $0.196^{* *}$ & -0.073 & $0.416^{* *}$ \\
\hline MCS & $\{193\}$ & $\{189\}$ & $\{190\}$ & $\{191\}$ & $\{191\}$ & $\{190\}$ \\
\hline SF-36 & $-0.351^{* *}$ & -0.062 & $-0.300^{* *}$ & $0.253^{* *}$ & $-0.150^{*}$ & $0.423^{* *}$ \\
\hline Total score & $\{190\}$ & $\{186\}$ & $\{187\}$ & $\{188\}$ & $\{189\}$ & $\{187\}$ \\
\hline EQ-5D & $-0.242^{* *}$ & 0.015 & $-0.193^{* *}$ & 0.086 & $-0.177^{*}$ & 0.085 \\
\hline VAS & $\{204\}$ & $\{200\}$ & $\{202\}$ & $\{202\}$ & $\{202\}$ & $\{199\}$ \\
\hline CLDQ & $-0.211^{* *}$ & -0.017 & -0.090 & 0.019 & -0.026 & $0.237^{* *}$ \\
\hline Full scale & $\{153\}$ & $\{153\}$ & $\{153\}$ & $\{153\}$ & $\{153\}$ & $\{153\}$ \\
\hline
\end{tabular}

${ }^{*} P<0.05$ and ${ }^{* *} p<0.01$ correlational relationship.

the risk of type II errors in our statistical analyses. Additionally, the small sample size also precluded us from further dividing our patients according to liver function level. Second, the study population was not fully representative of all patients with HB in Zhejiang Province. Finally, the study sample was somewhat heterogeneous in that participants were recruited from several hospitals in several cities, which can introduce noise into the results.

In conclusion, we documented diminished HRQoL levels in patients with $\mathrm{HB}$ in southeastern China. We observed particularly strong significant inverse associations of HRQoL with disease stage and income level in patients with $\mathrm{HB}$, as well as significant associations with older age, being without a marital partner, and education level. Based on these findings, it is our view that more social and medical supports are needed for patients with $\mathrm{HB}$ in southeastern China, particularly those with an advanced disease stage and low household income, to improve HRQoL in this population.

\section{Data Availability}

The data used in this article are available from the corresponding author upon reasonable request (ljli@zju.edu.cn).

\section{Conflicts of Interest}

The authors declare that they have no conflicts of interest.

\section{Authors' Contributions}

Ping Chen and Fen Zhang contributed equally to this study.

\section{Acknowledgments}

This work was supported by the 13th Five-Year National Science and Technology Major Project (2017ZX10105001003 and 2017ZX10302201-009) and Medical and Health Technology Program of Zhejiang Province (2020RC032).

\section{Supplementary Materials}

Supplementary Table 1: the laboratory parameters were obtained from medical records or the hospital database. The
$\mathrm{CHB}$ and $\mathrm{HB}$ cirrhosis groups were compared according to the baseline laboratory parameters. A normal distribution test indicated whether the data were parametric. Total bilirubin met normal distribution and was analyzed using Student's $t$-test. Other data were analyzed using Mann-Whitney $U$ tests to compare the two HB groups. Supplementary Figure 1: we briefly summarized the incorporation process of patients with $\mathrm{HB}$, including patients with $\mathrm{CHB}$ and $\mathrm{HB}$ cirrhosis. Three hundred potential patients with $\mathrm{HB}$ participated in this study. Among these 300 patients, 146 patients were excluded for not receiving antiviral therapy, leaving 164 patients with HB. Ten patients were excluded for returning incomplete questionnaires. (Supplementary Materials)

\section{References}

[1] T. Tu, J. M. Block, S. Wang, C. Cohen, and M. W. Douglas, "The lived experience of chronic hepatitis B: a broader view of its impacts and why we need a cure," Viruses, vol. 12, no. 5, 2020.

[2] WHO, Global Hepatitis Report 2017, WHO, Geneva, Switzerland, 2017, https://www.who.int/hepatitis/publications/ global-hepatitis-report2017/en/.

[3] S. Chen, J. Li, D. Wang, H. Fung, L.-Y. Wong, and L. Zhao, "The hepatitis B epidemic in China should receive more attention," The Lancet, vol. 391, no. 10130, p. 1572, 2018.

[4] H. Roberts, D. Kruszon-Moran, K. N. Ly et al., "Prevalence of chronic hepatitis B virus (HBV) infection in U.S. households: national health and nutrition examination survey (NHANES), 1988-2012," Hepatology, vol. 63, no. 2, pp. 388-397, 2016.

[5] A. Schweitzer, J. Horn, R. T. Mikolajczyk, G. Krause, and J. J. Ott, "Estimations of worldwide prevalence of chronic hepatitis B virus infection: a systematic review of data published between 1965 and 2013," The Lancet, vol. 386, no. 10003 , pp. 1546-1555, 2015.

[6] Y. Cui and J. Jia, "Update on epidemiology of hepatitis B and C in China," Journal of Gastroenterology and Hepatology, vol. 28, no. Suppl 1 7-10, 2013.

[7] G. L. Reynolds, H. H. Nguyen, S. Singh-Carlson, D. G. Fisher, A. Odell, and P. Xandre, "Application of the extended health control belief model to predict hepatitis $\mathrm{A}$ and $\mathrm{B}$ vaccinations," Public Health Nursing, vol. 33, no. 5, pp. 430-439, 2016.

[8] A. M. Biset and H. B. Adugna, "Hepatitis B vaccination status among health care workers in a tertiary hospital in Ethiopia," 
Hepatitis Research and Treatment, vol. 2017, Article ID 6470658, 8 pages, 2017.

[9] A. Schweitzer, M. K. Akmatov, and G. Krause, "Hepatitis B vaccination timing: results from demographic health surveys in 47 countries," Bulletin of the World Health Organization, vol. 95, no. 3, pp. 199-209, 2017.

[10] T. Vu, T. Le, A. Dang et al., "Socioeconomic vulnerability to depressive symptoms in patients with chronic hepatitis B," International Journal of Environmental Research and Public Health, vol. 16, no. 2, p. 255, 2019.

[11] B. Helbling, K. Overbeck, J.-J. Gonvers et al., "Host- rather than virus-related factors reduce health-related quality of life in hepatitis C virus infection," Gut, vol. 57, no. 11, pp. 1597-1603, 2008.

[12] R. Huang, H. Rao, J. Shang et al., "A cross-sectional assessment of health-related quality of life in Chinese patients with chronic hepatitis c virus infection with EQ-5D," Health and Quality of Life Outcomes, vol. 16, no. 1, p. 124, 2018.

[13] P. J. Thuluvath and Y. Savva, "Mental and physical healthrelated quality of life in patients with hepatitis $\mathrm{C}$ is related to baseline comorbidities and improves only marginally with hepatitis C cure," Clinical and Translational Gastroenterology, vol. 9, no. 4, p. 149, 2018.

[14] S. Bondini, J. Kallman, A. Dan et al., "Health-related quality of life in patients with chronic hepatitis B," Liver International, vol. 27, no. 8, pp. 1119-1125, 2007.

[15] E. T. Lam, C. L. Lam, C. L. Lai, M. F. Yuen, D. Y. Fong, and T. M. So, "Health-related quality of life of Southern Chinese with chronic hepatitis B infection," Health Qual Life Outcomes, vol. 7 52, 2009.

[16] S. C. Ong, B. Mak, M. O. Aung, S.-C. Li, and S.-G. Lim, "Health-related quality of life in chronic hepatitis B patients," Hepatology, vol. 47, no. 4, pp. 1108-1117, 2008.

[17] T. W. Lapinski, J. Stepaniuk, K. Tomasiewicz, D. Lebensztejn, M. Kulikowski, and R. Flisiak, "Effect of hepatitis B virus (HBV) infection on the course of pregnancy and newborns' health status," Journal of Clinical and Experimental Hepatology, vol. 1, no. 3, pp. 112-116, 2015.

[18] F. Guo and M. E. Wright, "Health status of Chinese women in Northern Ireland: SF-36 heath survey," Diversity in Health \& Social Care, vol. 2, no. 4, pp. 291-298, 2005.

[19] J. A. Boscarino, M. Lu, A. C. Moorman et al., "Predictors of poor mental and physical health status among patients with chronic hepatitis $\mathrm{C}$ infection: the chronic hepatitis cohort study (CHeCS)," Hepatology, vol. 61, no. 3, pp. 802-811, 2015.

[20] C. R. Dolder, J. P. Lacro, K. A. Warren, S. Golshan, D. O. Perkins, and D. V. Jeste, "Brief evaluation of medication influences and beliefs," Journal of Clinical Psychopharmacology, vol. 24, no. 4, pp. 404-409, 2004.

[21] A. R. Levy, K. V. Kowdley, U. Iloeje et al., "The impact of chronic hepatitis B on quality of life: a multinational study of utilities from infected and uninfected persons," Value in Health, vol. 11, no. 3, pp. 527-538, 2008.

[22] J. F. Gallegos-Orozco, A. P. Fuentes, J. G. Argueta et al., "Health-related quality of life and depression in patients with chronic hepatitis C," Archives of Medical Research, vol. 34, no. 2, pp. 124-129, 2003.

[23] J. R. Keltner, F. Vaida, R. J. Ellis et al., "Health-related quality of life 'well-being' in HIV distal neuropathic pain is more strongly associated with depression severity than with pain intensity," Psychosomatics, vol. 53, no. 4, pp. 380-386, 2012.

[24] E. S. Jang, Y. S. Kim, K.-A. Kim et al., "Factors associated with health-related quality of life in Korean patients with chronic hepatitis C infection using the SF-36 and EQ-5D," Gut and Liver, vol. 12, no. 4, pp. 440-448, 2018.

[25] S. C. Chang, S. S. Yang, C. C. Chang, C. C. Lin, Y. C. Chung, and T. C. Li, "Assessment of health-related quality of life in antiviral-treated Taiwanese chronic hepatitis $\mathrm{C}$ patients using SF-36 and CLDQ," Health Qual Life Outcomes, vol. 12 97, 2014.

[26] J. J. Gutteling, R. A. de Man, J. J. Busschbach, and A. S. Darlington, "Overview of research on health-related quality of life in patients with chronic liver disease," The Netherlands Journal of Medicine, vol. 65, no. 7, pp. 227-234, 2007.

[27] L. Gu, J. Xie, J. Long et al., "Epidemiology of major depressive disorder in mainland China: a systematic review," PLoS One, vol. 8, no. 6, Article ID e65356, 2013.

[28] Y. Liu, K. Tang, J. Long, and C. Zhao, "The association between hepatitis B self-awareness and depression: exploring the modifying effects of socio-economic factors," Journal of Viral Hepatitis, vol. 24, no. 4, pp. 330-336, 2017.

[29] A. Sobhonslidsuk, C. Silpakit, R. Kongsakon, P. Satitpornkul, C. Sripetch, and A. Khanthavit, "Factors influencing healthrelated quality of life in chronic liver disease," World Journal of Gastroenterology, vol. 12, no. 48, pp. 7786-7791, 2006.

[30] O. Parkash, R. Iqbal, F. Jafri, I. Azam, and W. Jafri, "Frequency of poor quality of life and predictors of health related quality of life in cirrhosis at a tertiary care hospital Pakistan," BMC Research Notes, vol. 5, no. 1, p. 446, 2012. 